\title{
Prevalence of Psoriatic Arthritis Patients Achieving Minimal Disease Activity in Real-world Studies and Randomized Clinical Trials: Systematic Review with Metaanalysis
}

\author{
Mariele Zardin-Moraes, André Luis Ferreira Azeredo da Silva, Carla Saldanha, Charles \\ Lubianca Kohem, Laura C. Coates (1D), Lilian Rodrigues Henrique, Penélope Esther Palominos (D), \\ and Rafael Mendonça da Silva Chakr (iD
}

\begin{abstract}
Objective. To estimate the frequency of patients with psoriatic arthritis (PsA) achieving minimal disease activity (MDA) status in real-world studies and randomized controlled trials (RCT).

Methods. A systematic literature search for 2009-2017 was performed in PubMed, Embase, Cochrane Library, and LILACS. Study selection and data extraction were performed by 2 independent researchers. Random-effects single-arm metaanalyses were performed and heterogeneity was assessed using $\mathrm{I}^{2}$.

Results. A total of 405 records were identified and 45 studies were analyzed: 39 (86.7\%) observational studies and $6(13.3 \%)$ RCT; they included 12,469 patients. The overall prevalence of MDA in cross-sectional studies was 35\% (95\% CI 30\%-41\%, $\mathrm{I}^{2}=94 \%$ ), varying from $17 \%$ (95\% CI 7\%-34\%) in patients taking synthetic disease-modifying antirheumatic drugs (DMARD) to $57 \%$ (95\% CI $41 \%-71 \%$ ) in those taking biological DMARD. Prevalence of MDA in cohort studies increased with longer followup time, ranging from $25 \%$ (95\% CI 15\%-40\%) with 3- to 4-month followup to $42 \%$ (95\% CI 38\%-45\%) with > 24-month followup. Patients with PsA receiving biological DMARD in a real-world context and RCT had similar prevalence of MDA at 6-month followup: $30 \%$ (95\% CI $\left.21 \%-41 \%, \mathrm{I}^{2}=85 \%\right)$ versus $32 \%\left(95 \%\right.$ CI $\left.26 \%-39 \%, \mathrm{I}^{2}=79 \%\right)$, respectively.

Conclusion. Patients with PsA included in real-world studies had similar prevalence of MDA compared to those in controlled clinical trials. This finding suggests that MDA is a useful treatment target for PsA in the real-world setting. (First Release April 15 2020; J Rheumatol 2020;47:839-46; doi:10.3899/jrheum.190677)
\end{abstract}

Key Indexing Terms:

PSORIATIC ARTHRITIS

\section{OBSERVATIONAL STUDIES \\ RANDOMIZED CONTROLLED TRIALS}

From the Rheumatology Department, Hospital de Clínicas de Porto Alegre, Universidade Federal do Rio Grande do Sul, Porto Alegre, Brazil; Nuffield Department of Orthopaedics, Rheumatology and Musculoskeletal Sciences, University of Oxford, Oxford, UK.

Supported by Fundo de Incentivo à Pesquisa e Eventos do Hospital de Clínicas de Porto Alegre.

M. Zardin-Moraes, MSc, Rheumatology Department, Hospital de Clínicas de Porto Alegre; A.L. Azeredo da Silva, PhD, Rheumatology Department, Hospital de Clínicas de Porto Alegre; C. Saldanha, MSc, Rheumatology Department, Hospital de Clínicas de Porto Alegre; C.L. Kohem, PhD, Rheumatology Department, Hospital de Clínicas de Porto Alegre; L.C. Coates, PhD, Nuffield Department of Orthopaedics, Rheumatology and Musculoskeletal Sciences, University of Oxford; L.R. Henrique, Rheumatology Department, Hospital de Clínicas de Porto Alegre; P.E. Palominos, PhD, Rheumatology Department, Hospital de Clínicas de Porto Alegre; R.M. Chakr, PhD, Rheumatology Department, Hospital de Clínicas de Porto Alegre. P.E. Palominos and R.M. Chakr contributed equally to this report.

Address correspondence to R.M. Chakr, Rheumatology Department,

Hospital de Clinicas de Porto Alegre, Ramiro Barcelos St. 2350, Porto

Alegre,Brazil.E-mail:rchakr@hcpa.edu.br

Accepted for publication September 18, 2019.
Psoriatic arthritis (PsA) is a chronic inflammatory disease that affects $20 \%-33 \%$ of individuals with psoriasis, and about 130 in every 100,000 individuals worldwide ${ }^{1,2}$. PsA has heterogeneous manifestations, affecting peripheral and axial joints, skin, nails, and entheses ${ }^{3}$.

Minimal disease activity (MDA) criteria have been recommended as a therapeutic target in $\mathrm{PsA}^{4,5}$. Patients are classified as achieving MDA if they fulfill 5 out of 7 outcome measures: tender joint count (TJC) $\leq 1$; swollen joint count $(\mathrm{SJC}) \leq 1$; Psoriasis Area and Severity Index $\leq 1$ or body surface area $\leq 3$; patient pain on visual analog scale (pain VAS) score $\leq 15$; patient global disease activity (global VAS) score $\leq 20$; Health Assessment Questionnaire score $\leq 0.5$; and tender entheseal points $\leq 1^{6}$.

The prevalence of MDA has been assessed in several trials and its frequency varies according to the study design, drug used, and time of evaluation. According to a recent publication, the frequency of MDA in randomized controlled trials (RCT)

Personal non-commercial use only. The Journal of Rheumatology Copyright $($ C 2020. All rights reserved. 
varies from $24 \%$ to $52 \%$ with tumor necrosis factor (TNF) inhibitor therapy and from $14 \%$ to $41 \%$ with secukinumab therapy, and from $40 \%$ to $64 \%$ in observational studies ${ }^{7}$.

Participants in RCT usually present higher levels of disease activity, fewer comorbidities ${ }^{8}$, and better adherence rates to therapy than patients analyzed in observational studies $^{9}$. In real life, factors such as poor adherence and restricted access to drugs preclude the achievement of MDA status in a treat-to-target strategy ${ }^{10}$.

The aim of our investigation was to analyze the frequency of patients with PsA achieving MDA status in real-world studies and RCT.

\section{MATERIALS AND METHODS}

Our study is a systematic review with metaanalysis of observational and interventional studies reporting MDA in patients with PsA.

Study protocol. The protocol for this systematic review is found in the international prospective register of systematic reviews (PROSPERO), record number CRD42016050502.

An extensive literature search was performed in April 2017 in PubMed, Embase, Cochrane, and LILACS with no limits or filters. The following search strategies were used.

PubMed and Cochrane: (("minimal disease activity" OR "minimal disease activities" OR "MDA")) AND "Arthritis, Psoriatic" [MESH] OR "Psoriasis, Arthritic" OR "Arthritic Psoriasis" OR "Psoriatic Arthritis" OR "Psoriasis Arthropathica" OR "Psoriatic Arthropathy" OR "Arthropathies, Psoriatic" OR "Arthropathy, Psoriatic" OR "Psoriatic Arthropathies" OR "Spondylarthropathies" [MESH] OR "Marie-Strumpell Spondylitis" OR "Marie Strumpell Spondylitis" OR "Spondylitis, Marie-Strumpell" OR "Spondyloarthropathy" OR "Spondyloarthropathies" OR "Bechterew Syndrome" OR "Syndrome, Bechterew" OR "Spondylarthropathy" OR "Spondylarthritis" [MESH] OR "Spondylarthritides" OR "Spinal Arthritis" OR "Spinal Arthritides" OR "Arthritis, Spinal”).

Embase: 'minimal disease activity' OR 'minimal disease activities' OR 'MDA' AND 'psoriatic arthritis'/exp OR 'psoriatic arthritis' OR 'alibertbazin disease' OR 'arthritis psoriatica' OR 'arthritis, psoriatic' OR 'arthritis, psoriasis' OR ‘arthritis, psoriatic' OR 'arthropathic psoriasis' OR 'arthropathy, psoriatic' OR 'disease, alibertbazin' OR 'polyarthritis, psoriatic' OR 'psoriasis arthropathica' OR 'psoriasis pustulosaarthropathica' OR 'psoriasis, arthritis' OR 'psoriatic arthropathy' OR 'psoriatic polyarthritis' OR 'psoriatic rheumatism' OR 'psoriatic rheumatoid arthritis' OR 'rheumatoid arthritis, psoriatic'.

LILACS: Minimal AND disease AND activity AND psoriatic arthritis.

An active search was carried out for abstracts presented from 2009 to 2017 in the European League Against Rheumatism (EULAR) Annual European Congress of Rheumatology, the American College of Rheumatology Annual Meeting, the World Psoriasis and Psoriatic Arthritis Conference, the International Congress of Spondyloarthropathies, and the Brazilian Congress of Rheumatology.

Inclusion criteria were original studies reporting the prevalence of MDA status among adult patients with PsA.

Exclusion criteria were (1) duplicates (in case of duplicates, the most complete publication was included, e.g., in case of abstracts and original articles reporting the same results, only the complete original article results were considered); (2) review articles, letters to the editor, case reports; (3) articles not describing MDA according to Coates and Helliwell ${ }^{6}$, e.g., studies that evaluated number of swollen joints other than 66 and tender joints other than 68 , studies that did not evaluate enthesitis, etc.; and (4) articles reporting only the prevalence of sustained MDA.

Selection of studies: 2 reviewers (MZ and CK) independently selected the articles by title and abstract. In the next step, 2 researchers (MZ and PP) independently selected the articles based on full text. Disagreements were resolved by consensus.
Data collection. The following data were extracted: first author, year of publication, country where study was conducted, duration of study, type of publication (original article vs abstract presented in congress), study design (RCT, cohort, cross-sectional), total number of patients included in study, total number and relative frequency of women included in study, mean age of population, PsA duration, number of comorbidities, time of evaluation, number and percentage of participants achieving MDA status, and mean and SD of each component of MDA criteria. The type of treatment was assessed and classified as biological disease-modifying antirheumatic drug (bDMARD) monotherapy, synthetic DMARD (sDMARD) monotherapy, combined bDMARD + sDMARD therapy, nonsteroidal antiinflammatory drugs (NSAID), and unspecified treatment.

These data were independently extracted from articles by 2 reviewers (MZ and CS), and disagreements were resolved by consensus.

Statistical analyses. To analyze the prevalence of MDA in observational trials, a single-arm metaanalysis was performed, grouping both cohort and cross-sectional studies and including all observational trials irrespective of treatment evaluated. The final followup time of each cohort study was considered in the analysis.

Subsequently, the studies were separated according to design: a single-arm metaanalysis including only cross-sectional studies was performed and another analysis including only cohort studies according to followup time (baseline, 3-4 mos, 6-8 mos, 12-13 mos, and 24-60 mos) was conducted.

$R C T$. Single-arm metaanalysis was performed to estimate the frequency of MDA in RCT, considering maximum followup time and including all RCT irrespective of treatment evaluated.

A single-arm metaanalysis was also performed to analyze the frequency of MDA in patients treated with bDMARD in real-world studies and RCT at the 6-month followup.

The results of the metaanalyses were represented by forest plots. The $\mathrm{I}^{2}$ index was used to assess heterogeneity. When relevant heterogeneity was found ( $\mathrm{I}^{2}$ index $\geq 50 \%$ ), the results from the random-effects model were shown, and in cases in which $\mathrm{I}^{2}$ was $<50 \%$, the fixed-effects model was used $^{11}$.

\section{RESULTS}

A total of 405 records were identified from databases. Of them, 274 were excluded by title and abstract and 96 studies were excluded after full-text analysis. Ten abstracts were identified. The 45 eligible studies included in the final analysis are listed in Table 1. An illustration summarizing the selection of studies is given in Figure 1.

The 45 selected studies included 12,469 patients, mean age $51.0(\mathrm{SD} \pm 3.3)$ years, mostly men $(\mathrm{n}=6386,51.2 \%)$, with a mean PsA disease duration of $8.1(\mathrm{SD} \pm 3.6)$ years. Thirty-nine $(86.7 \%)$ were real-world studies and only 6 (13.3\%) were RCT. Observational studies, on average, had larger populations and longer followup times compared to RCT. Among the 39 observational studies, 19 (48.7\%) were carried out in Europe and 10 (25.6\%) in North America, and $37(94.9 \%)$ were conducted in university hospitals.

The characteristics of studies included in the analysis are shown in Table 2.

The assessment of bias is described in Supplementary Tables 1-3, available from the authors on request.

Frequency of MDA in real-life studies. The frequency of PsA patients achieving MDA status in real-world studies was $37 \%\left(95 \%\right.$ CI $\left.34 \%-41 \%, \mathrm{I}^{2}=93 \%\right)$ when both crosssectional and cohort studies were grouped and all types 
Table 1. The 45 studies eligible for inclusion in our analysis comprised data for 12,469 patients.

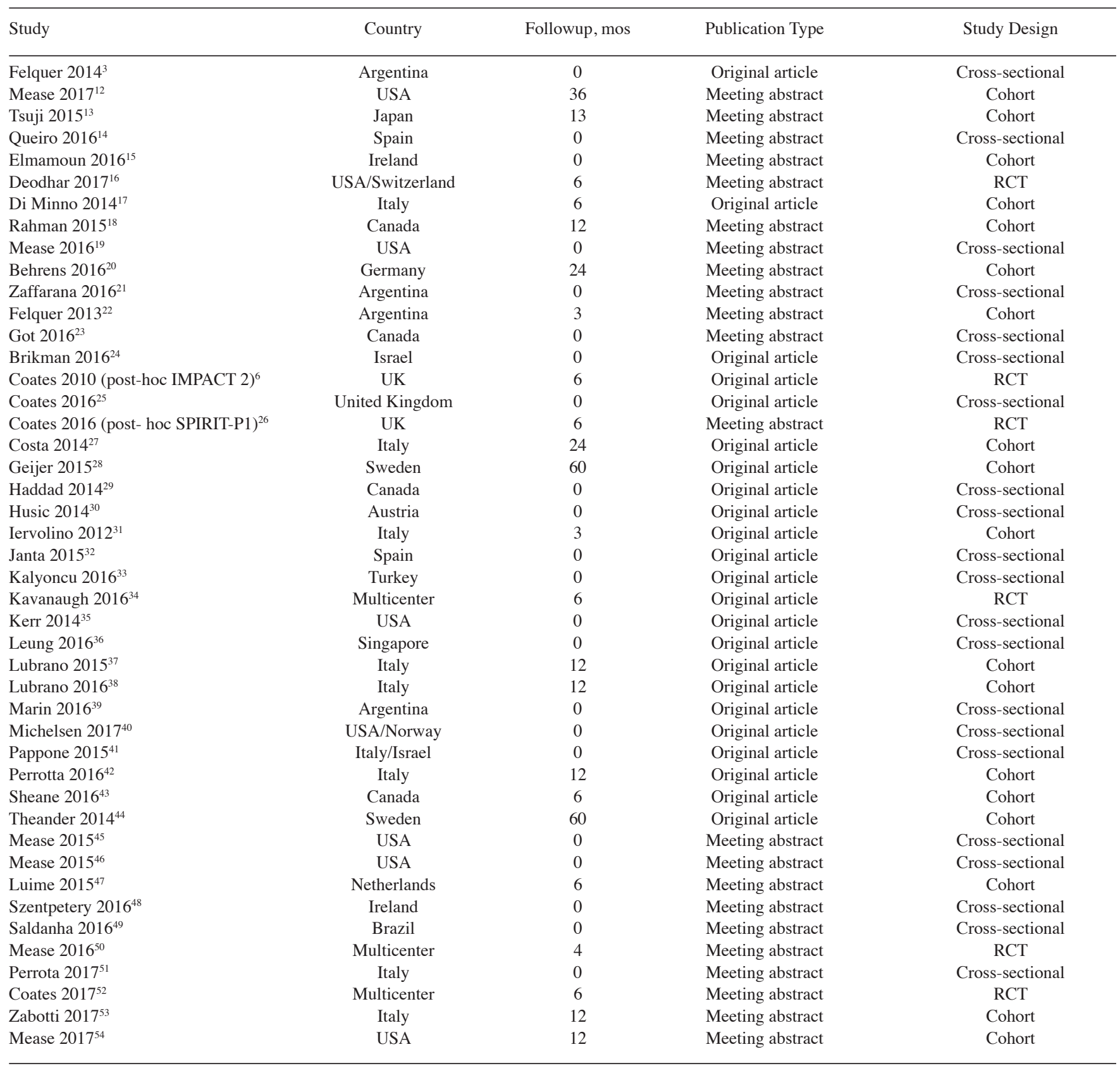

RCT: randomized controlled trial.

of treatment were considered. When only cross-sectional studies were considered $(n=22)$, the overall frequency of MDA was 35\% (95\% CI 30\%-41\%, $\mathrm{I}^{2}=94 \%$; Figure 2), varying from $17 \%(95 \%$ CI $7 \%-34 \%)$ in the sole study that specified that patients were taking sDMARD to $57 \%(95 \%$ CI $41 \%-71 \%, \mathrm{I}^{2}=87 \%$ ) in studies evaluating patients in use of anti-TNF therapy. Assessment of the prevalence of MDA in a real-world context in different treatment subgroups was restricted because $81.8 \%(n=18)$ of cross-sectional studies did not specify the patients' current therapy.
In cohort studies, the frequency of patients with MDA increased with longer followup time, varying from $25 \%$ $\left(95 \%\right.$ CI $\left.15 \%-40 \%, \mathrm{I}^{2}=87 \%\right)$ when MDA was evaluated at $3-4$ months, to $30 \%\left(95 \%\right.$ CI $\left.21 \%-42 \%, \mathrm{I}^{2}=94 \%\right)$ at 6-8 months, $42 \%\left(95 \%\right.$ CI $\left.39 \%-46 \%, \mathrm{I}^{2}=68 \%\right)$ at $12-13$ months, and $42 \%\left(95 \%\right.$ CI $\left.38 \%-45 \%, \mathrm{I}^{2}=64 \%\right)$ in studies with $\geq 24$ months followup (Supplementary Figure 1, available from the authors on request). When patients treated with biological drugs in cohort studies were compared to those treated with bDMARD in RCT, the frequency of MDA

Personal non-commercial use only. The Journal of Rheumatology Copyright @ 2020 . All rights reserved. 


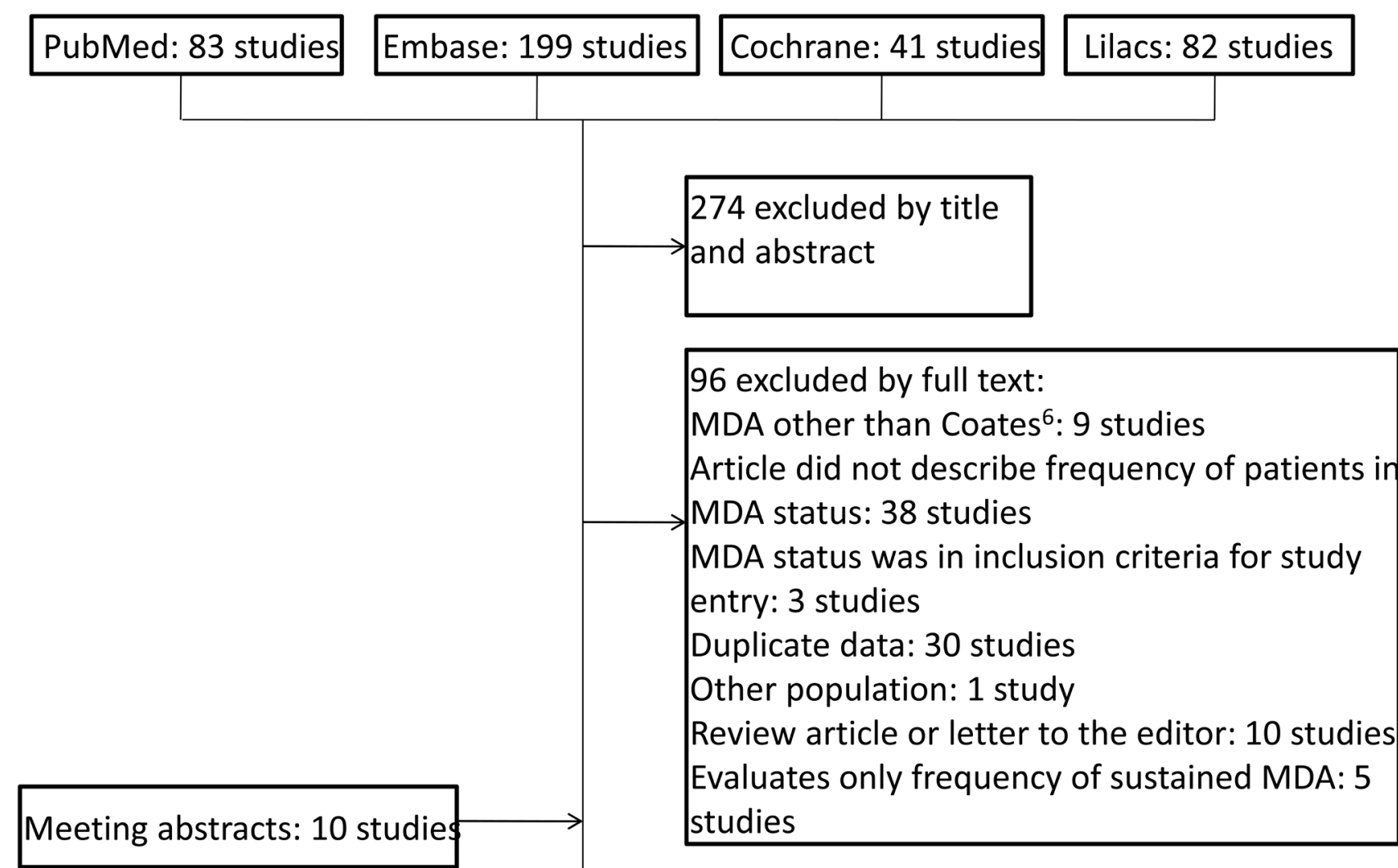

Total: 45 selected studies

Figure 1. A summary of the selection of studies. MDA: minimal disease activity.

Table 2. Characteristics of studies included in the analysis.

\begin{tabular}{|c|c|c|c|}
\hline Characteristic & All Articles, $n=45$ & Real-world Studies, $\mathrm{n}=39$ & $\mathrm{RCT}, \mathrm{n}=6$ \\
\hline Total no. patients & 12,469 & 11,254 & 1215 \\
\hline Female, n $(\%)$ & $6083(48.8)$ & $5588(49.6)$ & $495(40.7)^{*}$ \\
\hline Age, yrs, mean \pm SD & $51.0 \pm 3.3$ & $51.2 \pm 3.3$ & $49.2 \pm 2.8^{*}$ \\
\hline Disease duration, yrs, mean $\pm \mathrm{SD}$ & $8.1 \pm 3.6$ & $7.9 \pm 4.0$ & $7.9 \pm 1.8^{*}$ \\
\hline $\begin{array}{l}\text { Duration of followup, mos, mean } \pm \text { SD } \\
\text { Treatment assessed, } \mathrm{n}(\%)\end{array}$ & $7.5 \pm 13.5$ & $7.8 \pm 14.6$ & $5.7 \pm 0.7$ \\
\hline Combined therapy bDMARD + sDMARD & $12(26.7)$ & $12(30.8)$ & $0(0.0)$ \\
\hline bDMARD monotherapy & $17(37.8)$ & $11(28.2)$ & $6(100)$ \\
\hline sDMARD monotherapy & $1(2.2)$ & $1(2.6)$ & $0(0.0)$ \\
\hline Treatment not specified & $15(33.3)$ & $15(38.5)$ & $0(0.0)$ \\
\hline
\end{tabular}

*Posthoc analyses. RCT: randomized controlled trial; bDMARD: biological disease-modifying antirheumatic drug; sDMARD: synthetic DMARD.

was similar at 6 months' followup: $30 \%(95 \%$ CI $21 \%-41 \%$, $\left.\mathrm{I}^{2}=85 \%\right)$ versus $32 \%\left(95 \%\right.$ CI $\left.26 \%-39 \%, \mathrm{I}^{2}=79 \%\right)$, respectively (Supplementary Figure 3).

Frequency of MDA in RCT. The 6 titles included in the analysis evaluated $7 \mathrm{RCT}$ (1 report described the results of 2 RCT) and all were trials evaluating biological therapy.
When all biological therapies (anti-TNF, anti-IL-17, and anti-IL-12/23) were grouped, the prevalence of PsA patients with MDA status was $32 \%\left(95 \%\right.$ CI $\left.27 \%-38 \%, \mathrm{I}^{2}=78 \%\right)$ in the biological therapy arm, compared with only $9 \%$ of patients with MDA in the placebo group (95\% CI 5\%-15\%, $\mathrm{I}^{2}=78 \%$ ) at roughly 6 months' followup (Figure 3 ). The 
Study

Felquer MLA et al 2014

Haddad A et al 2014

Husic R et al 2014

Kerr G et al 2014

Janta I et al 2015

Pappone N et al 2015

Mease PJ et al 2015

Mease PJ, Karki C et al 2015

Queiro R et al 2016

Mease PJ et al 2016

Zaffarana C et al 2016

Got M et al 2016

Brikman S et al 2016

Coates LC et al 2016

Kalyoncu U et al 2016

Leung $Y Y$ et al 2016

Marin J et al 2016

Szentpetery A et al 2016

Saldanha C, Zardin M et al 2016

Michelsen B et al 2017

Perrotta FM et al 2017

Perrotta FM et al 2017

Fixed effect model

Random effects model

Heterogeneity: $l^{2}=94 \%, \tau^{2}=0.2451, p<0.01$
Events Total

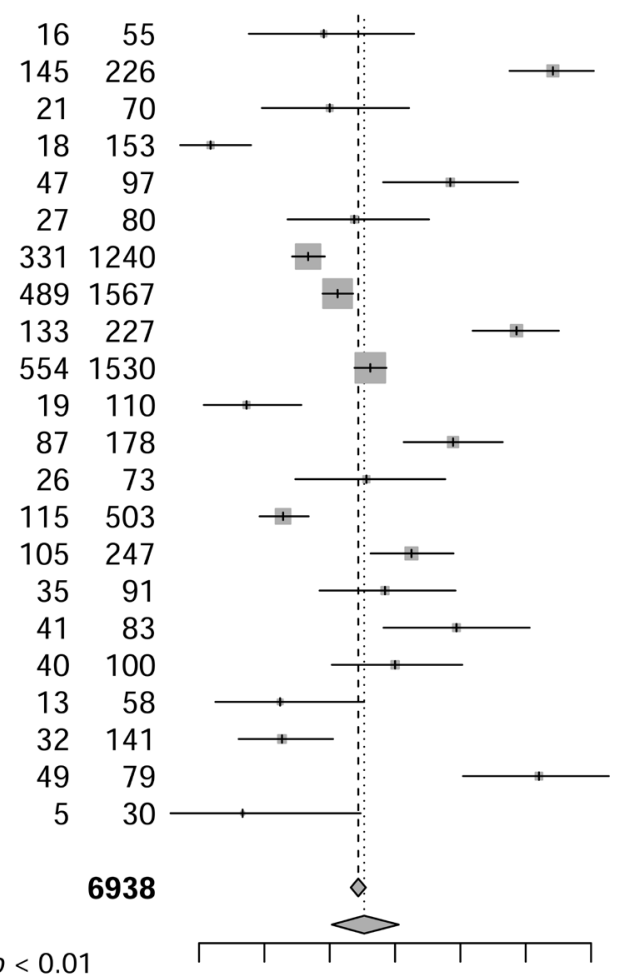

Proportion

95\%-CI

$0.29[0.18 ; 0.43]$

$0.27[0.24 ; 0.29] \quad 16.5 \%$
$0.64[0.58 ; 0.70]$

$0.30[0.20 ; 0.42]$

$0.12[0.07 ; 0.18]$

$0.48[0.38 ; 0.59]$

$0.34[0.24 ; 0.45]$

$0.31 \quad[0.29 ; 0.34] \quad 22.8 \%$

$0.59[0.52 ; 0.65] \quad 3.7 \%$

$0.36[0.34 ; 0.39] \quad 24.0 \%$

$0.17[0.11 ; 0.26] \quad 1.1 \%$

$0.49[0.41 ; 0.56] \quad 3.0 \%$

$0.36[0.25 ; 0.48] \quad 1.1 \%$

$0.23[0.19 ; 0.27] \quad 6.0 \%$

$0.43[0.36 ; 0.49] \quad 4.1 \%$

$0.38[0.28 ; 0.49] \quad 1.5 \%$

$0.49[0.38 ; 0.61] \quad 1.4 \%$

$0.40[0.30 ; 0.50] \quad 1.6 \%$

$0.22[0.13 ; 0.35] \quad 0.7 \%$

$0.23[0.16 ; 0.31] \quad 1.7 \%$

$0.62[0.50 ; 0.73] \quad 1.3 \%$

$0.17[0.06 ; 0.35] \quad 0.3 \%$

$0.34[0.33 ; 0.36] 100.0 \%$

$0.35[0.30 ; 0.41]$

\section{$.8 \%$}

$1.5 \%$

$.1 \%$

$.6 \%$

$2 \%$

\%

$\%$

$5.3 \%$

$5.3 \%$

$5.0 \%$

$5.3 \%$

$4.3 \%$

$4.9 \%$

$4.3 \%$

$5.1 \%$

$5.0 \%$

$4.5 \%$

$4.5 \%$

$4.6 \%$

$3.8 \%$

$4.6 \%$

$4.4 \%$

$2.7 \%$

$\begin{array}{lllllll}0.1 & 0.2 & 0.3 & 0.4 & 0.5 & 0.6 & 0.7\end{array}$

Figure 2. The overall frequency of minimal disease activity was $35 \%$ when cross-sectional studies only were considered $(n=22)$.

prevalence of MDA was similar across studies of bDMARD therapy: $30 \%\left(95 \%\right.$ CI $\left.27 \%-51 \%, \mathrm{I}^{2}=83 \%\right)$ in studies evaluating anti-TNF therapy, $29 \%$ (95\% CI $23 \%-36 \%$, $\mathrm{I}^{2}=72 \%$ ) in those evaluating anti-interleukin (IL) 17 drugs, and $23 \%$ (95\% CI 16\%-32\%, $\mathrm{I}^{2}$ not applicable) in the sole study evaluating anti-IL-12/23 therapy included in the analysis (Supplementary Figure 2, available from the authors on request).

\section{DISCUSSION}

To our knowledge, this is the first systematic literature review with a metaanalysis to compare performance of MDA in RCT and observational studies of patients with PsA. Knowing how MDA performs in RCT and real-life scenarios may contribute to improving its feasibility. Our work showed that about one-third of patients with PsA evaluated in cross-sectional studies were in a state of MDA, with this value varying from $17 \%$ (95\% CI $7 \%-34 \%)$ with the use of sDMARD to $57 \%$ (95\% CI $41 \%-71 \%$ ) with bDMARD. In addition, the longer the followup time in real-world studies, the higher the prevalence of patients achieving MDA. Patients receiving bDMARD had similar prevalence of MDA status at 6 months' followup in RCT and observational studies.

The initial hypothesis predicted a higher frequency of
MDA in RCT due to better adherence to therapy, because barriers to acquisition of medication and cost of therapy can be problems in daily life. However, even in RCT, adherence rates can range from $43 \%$ to $78 \%$ among patients receiving treatment for chronic diseases. The factors that are related to lower adherence in RCT and real-world studies include psychiatric comorbidities, cognitive impairment, inadequate followup, adverse effects of medication, poor physician-patient relationship, missed appointments, and treatment complexity ${ }^{9}$.

Patients included in RCT usually have higher levels of disease activity than those in real-world studies ${ }^{55}$, and this may limit the final outcomes of the studies $^{8}$, preventing patients from achieving MDA.

On the other hand, in real-world studies we expect lower response rates because of lower adherence, but in this case, most of the patients have milder disease. Thus we expect lower response rates because of lower adherence to therapy but higher response because disease is milder and treatment is open-label.

In our investigation, RCT and real-world studies had similar frequencies of MDA probably because the selected observational studies were carried out mainly in university hospitals located in developed countries, where patients with PsA have adequate education levels, receive a high standard

Personal non-commercial use only. The Journal of Rheumatology Copyright (c) 2020. All rights reserved. 
GRUPO = 1

Coates LC, 2010 (post hoc IMPACT) Anti-TNF

Coates LC, 2010 (post hoc IMPACT 2) Anti-TNF

Mease P, 2016 (post hoc FUTURE 2) IL-17

Coates LC, 2016 (SPIRIT-P1) IL-17

Kavanaugh A, 2016 (GO REVEAL) Anti-TNF

Coates LC, 2016 (SPIRIT-P1) Anti-TNF

Coates LC, 2017 (post hoc SPIRIT-P2) IL-17

Deodhar A, 2017 IL-23

Fixed effect model

Random effects model

Heterogeneity: $I^{2}=78 \%, \tau^{2}=0.1126, p<0.01$

GRUPO $=2$

Coates LC, 2010 (post hoc IMPACT) Placebo

Coates LC, 2010 (post hoc IMPACT 2) Placebo

Mease P, 2016 (post hoc FUTURE 2) Placebo

Coates LC, 2016 (SPIRIT-P1) Placebo

Kavanaugh A, 2016 (GO REVEAL) Placebo

Coates LC, 2017 (post hoc SPIRIT-P2) Placebo

Deodhar A, 2017 Placebo

Fixed effect model

Random effects model

Heterogeneity: $I^{2}=73 \%, \tau^{2}=0.4177, p<0.01$

Fixed effect model

Random effects model

Heterogeneity: $I^{2}=87 \%, \tau^{2}=0.3604, p<0.01$

$\begin{array}{rr}15 & 31 \\ 40 & 77 \\ 50 & 197 \\ 75 & 210 \\ 80 & 285 \\ 32 & 101 \\ 63 & 245 \\ 23 & 100 \\ & 1246\end{array}$

7
7
0
5
1
5
6

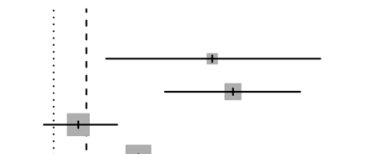

$0.48[0.30 ; 0.67]-2.5 \%$

$0.52[0.40 ; 0.63] \quad 6.3 \%$

$0.25[0.19 ; 0.32] 12.2 \%$

$0.36[0.29 ; 0.43] \quad 15.8 \%$

$0.28[0.23 ; 0.34] 18.9 \%$

$0.32[0.23 ; 0.42] \quad 7.2 \%$

$0.26[0.20 ; 0.32] 15.4 \%$

$0.23[0.15 ; 0.32] \quad 5.8 \%$

$0.31[0.28 ; 0.33] \quad 84.2 \%$

$0.32[0.27 ; 0.38] \quad--$

$6.5 \%$

$7.7 \%$

$8.2 \%$

$8.3 \%$

$8.4 \%$

$7.8 \%$

$8.3 \%$

$7.6 \%$

$--$

$62.8 \%$

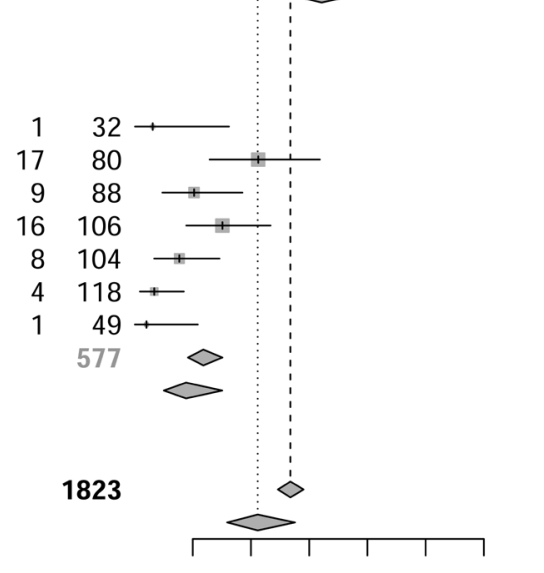

$\begin{array}{llllll}0.1 & 0.2 & 0.3 & 0.4 & 0.5 & 0.6\end{array}$
$0.03[0.00 ; 0.16] \quad 0.3 \%$

$0.21[0.13 ; 0.32] \quad 4.4 \%$

$0.10[0.05 ; 0.19] \quad 2.7 \%$

$0.15[0.09 ; 0.23] \quad 4.5 \%$

$0.08[0.03 ; 0.15] \quad 2.4 \%$

$0.03[0.01 ; 0.08] \quad 1.3 \%$

$0.02[0.00 ; 0.11] \quad 0.3 \%$

$0.12[0.09 ; 0.15] 15.8 \%$

$0.09[0.05 ; 0.15] \quad--$

$2.3 \%$

$7.3 \%$

$6.5 \%$

$7.3 \%$

$6.4 \%$

$5.1 \%$

$2.3 \%$

$--$

$37.2 \%$

$0.27[0.25 ; 0.29] 100.0 \%$

$0.21[0.16 ; 0.28] \quad--100.0 \%$

Figure 3. When all biological therapies (anti-TNF, anti-IL-17, and anti-IL-12/23) were grouped, the prevalence of PsA patients with minimal disease activity (MDA) status was 32\% in the biological therapy arm, compared to only $9 \%$ of patients with MDA in the placebo group at 6 months of followup. TNF: tumor necrosis factor; IL: interleukin; PsA: psoriatic arthritis.

of care, and have easy access to DMARD. In underdeveloped countries, in contrast, restricted access to DMARD and low education levels leading to poor understanding of medication instructions were barriers to achieving MDA in the real-world context ${ }^{10}$.

Our results were similar to those from a study ${ }^{12}$ that described the frequency of MDA in RCT varying from $24 \%$ to $52 \%$ in patients receiving anti-TNF therapy, and from $23 \%$ to $28 \%$ in those receiving secukinumab (at 16 weeks). In observational studies, the frequency of MDA was $44 \%-64 \%$ at 12 months, and $40 \%$ at the 5-year followup ${ }^{7}$. Another study described that the frequency of MDA in real-life studies ranged from $15 \%$ to $64 \%{ }^{49}$.

Limitations of our report are the great heterogeneity regarding characteristics of patients, current therapies, and followup times in the studies we investigated. There was greater amplitude in effect sizes among observational studies compared to RCT, suggesting that the groups included in the real-life studies were more heterogeneous. In addition, the majority of real-life studies did not specify the current treatment of patients or describe the MDA in the different treatment subgroups. Further, in many studies assessment of MDA was not the primary outcome and its prevalence was described only in posthoc analysis.
A priori observational studies are expected to have greater heterogeneity owing to the role of uncontrolled factors influencing achievement of MDA. The lack of a standardized treatment protocol within the same study and across various scenarios is a key factor explaining the different results. Also, in cohort and case-control studies, missing data may significantly affect the outcomes and no imputation is usually reported.

We acknowledge that in this case clinical heterogeneity should be considered a limitation in the lack of statistical homogeneity. Variances in inclusion criteria could affect achievement of MDA, even with similar treatment strategy protocols. This is particularly relevant in RCT. For example, in the IMPACT trial, 5 or more swollen/tender joints were considered as inclusion criteria, whereas in the FUTURE-2 and SPIRIT-P1 studies, 3 or more tender/swollen joints were required for inclusion. As well, previous treatments differed among studies. For instance, in FUTURE-2, participants could have failed NSAID, synthetic DMARD, or TNF inhibitors, whereas in SPIRIT-P1, subjects were required to be TNF inhibitor-naive, and in SPIRIT-P2 they were included only after an inadequate response to TNF inhibitor therapy.

We observed that patients with PsA included in real-world studies reported recently had similar prevalence of MDA

Personal non-commercial use only. The Journal of Rheumatology Copyright @ 2020 . All rights reserved. 
compared to those participating in controlled clinical trials. This finding suggests that MDA is a useful treatment target for PsA in a real-world context.

\section{REFERENCES}

1. Alinaghi F, Calov M, Kristensen LE, Gladman DD, Coates LC, Jullien D, et al. Prevalence of psoriatic arthritis in patients with psoriasis: a systematic review and meta-analysis of observational and clinical studies. J Am Acad Dermatol 2019;80:251-65.

2. Scotti L, Franchi M, Marchesoni A, Corrao G. Prevalence and incidence of psoriatic arthritis: a systematic review and meta-analysis. Semin Arthritis Rheum 2018;48:28-34.

3. Acosta Felquer ML, Ferreyra Garrott L, Marin J, Catay E, Scolnik $\mathrm{M}$, Scaglioni V, et al. Remission criteria and activity indices in psoriatic arthritis. Clin Rheumatol 2014;33:1323-30.

4. Gossec L, Smolen JS, Ramiro S, de Wit M, Cutolo M, Dougados M, et al. European League Against Rheumatism (EULAR) recommendations for the management of psoriatic arthritis with pharmacological therapies: 2015 update. Ann Rheum Dis 2016;75:499-510

5. Coates LC, FitzGerald O, Merola JF, Smolen J, van Mens LJJ, Bertheussen H, et al. Group for Research and Assessment of Psoriasis and Psoriatic Arthritis/Outcome Measures in Rheumatology consensus-based recommendations and research agenda for use of composite measures and treatment targets in psoriatic arthritis. Arthritis Rheumatol 2018;70:345-55.

6. Coates LC, Helliwell PS. Validation of minimal disease activity criteria for psoriatic arthritis using interventional trial data. Arthritis Care Res 2010;62:965-9.

7. Gossec L, McGonagle D, Korotaeva T, Lubrano E, de Miguel E, Ostergaard M, et al. Minimal disease activity as a treatment target in psoriatic arthritis: a review of the literature. J Rheumatol 2018; 45:6-13.

8. Monti S, Grosso V, Todoerti M, Caporali R. Randomized controlled trials and real-world data: differences and similarities to untangle literature data. Rheumatology 2018;57:vii54-8.

9. Osterberg L, Blaschke T. Adherence to medication. N Engl J Med 2005;353:487-97.

10. Ferreira M, Kohem C, Xavier R, Abegg E, Martins OS, Resmini $\mathrm{MB}$, et al. Treating psoriatic arthritis to target: discordance between physicians and patients' assessment, non-adherence and restricted access to drugs precluded therapy escalation in a real-world cohort. Clin Rheumatol 2019;38:961-68.

11. Serghiou S, Goodman S. Random-effects meta-analysis summarizing evidence with caveats. JAMA 2019;321:301-2.

12. Mease P, Palmer J, Litman H, Karki C, Greenberg J. Impact of nail psoriasis on clinical presentation of psoriatic arthritis - descriptive analysis From the Corrona Psoriatic Arthritis/Spondyloarthritis (PsA/SpA) Registry. J Am Acad Dermatol 2017;76:AB405.

13. Tsuji S, Higashiyama M, Tomita T, Matsui M, Tsuboi H, Hashimoto S. Predictors of minimal disease activity in patients treated with adalimumab for 52 weeks in clinical practice. Ann Rheum Dis 2015;74:871.

14. Queiro R, Cañete JD, Torre JC, Román-Ivorra JA, Sanz J, Montilla $\mathrm{C}$, et al. Study of prevalence and predictors of minimal disease activity (MDA) state in a Spanish population with psoriatic arthritis. Maaps Study. Poster presentation. Ann Rheum Dis 2016;75:344.

15. Elmamoun M, Szentpetery P, Gallagher P, FitzGerald O. Measuring outcome in psoriatic arthritis (MOPSA), a new-based tool for assessment of psoriatic arthritis showing initiation of treatment change in patients achieving minimal disease activity. Ann Rheum Dis 2016;75:606.

16. Deodhar A, Gottlieb A, Boehncke WH, Dong B, Wang Y, Barchuk $\mathrm{W}$, et al. Efficacy and safety results of guselkumab, an anti-IL23 monoclonal antibody, in patients with active psoriatic arthritis over 24 weeks: a phase $2 \mathrm{~A}$, randomized, double-blind,

placebo-controlled study. Ann Rheum Dis 2017;76 Suppl 2:142-3.

17. Di Minno MND, Iervolino S, Peluso R, Di Minno A, Ambrosino P, Scarpa R. Hemostatic and fibrinolytic changes are related to inflammatory conditions in patients with psoriatic arthritis - effect of different treatments. J Rheumatol 2014;41:714-22.

18. Rahman P, Zummer M, Bensen WG, Fortin I, Sholter D, Baker MF, et al. Effectiveness and safety of golimumab in the treatment of psoriatic arthritis over a 12 month period. Arthritis Rheum 2015;67:2866.

19. Mease PJ, Karki C, Liu M, Kavanaugh A, Pandurengan R, Ritchlin $\mathrm{CT}$, et al. Influence of axial involvement on clinical characteristics of psoriatic arthritis - descriptive analysis from the Corrona Psoriatic Arthritis/Spondyloarthritis (PsA/SpA) Registry [abstract]. Arthritis Rheum 2016;68 Suppl 10:1683.

20. Behrens F, Koehm M, Schwaneck EC, Schmalzing M, Gnann H. Minimal disease activity is a stable measure of therapeutic response in psoriatic arthritis patients receiving treatment with adalimumab. Arthritis Rheum 2016;68:1696

21. Zaffarana C, Yanzi JG, Cerda OL, Landi M, Schneeberger E, Citera G. Prevalence of obesity in patients with psoriatic arthritis and its impact on the severity of the disease. Arthritis Rheumatol 2016;68:3715-16

22. Felquer MLA, Ruta S, Rosa J, Navarta DA, Saucedo C, Garcia-Monaco R, et al. Responsiveness to change of a global ultrasound assessment score in psoriatic arthritis patients. Arthritis Rheum 2013;65:S129-30.

23. Got M, Li S, Perruccio AV, Gladman DD, Chandran V. Treating psoriatic arthritis (PsA) to target: defining Psoriatic Arthritis Disease Activity Score (PASDAS) that reflects disease activity in PsA. Arthritis Rheum 2016;68:1700.

24. Brikman S, Furer V, Wollman J, Borok S, Matz H, Polachek A, et al. The effect of the presence of fibromyalgia on common clinical disease activity indices in patients with psoriatic arthritis: a cross-sectional study. J Rheumatol 2016;43:1749-54.

25. Coates LC, Helliwell PS. Defining low disease activity states in psoriatic arthritis using novel composite disease instruments. J Rheumatol 2016;43:371-5.

26. Coates LC, Husni ME, Shuler CL, Carlier H, Lin C, Mou J, et al. Ixekizumab provides sustained improvement up to 52 weeks of disease activity as assessed by composite measure scores in biologic disease-modifying antirheumatic drug (bDMARD)-naive patients with active psoriatic arthritis. Ann Rheum Dis 2016;75:349.

27. Costa L, Caso F, Ramonda R, Del Puente A, Cantarini L, Darda MA, et al. Metabolic syndrome and its relationship with the achievement of minimal disease activity state in psoriatic arthritis patients: an observational study. Immunol Res 2015;61:147-53.

28. Geijer M, Lindqvist U, Husmark T, Alenius GM, Larsson PT, Teleman A, et al. The Swedish early psoriatic arthritis registry 5-year followup: substantial radiographic progression mainly in men with high disease activity and development of dactylitis. J Rheumatol 2015;42:2110-7.

29. Haddad A, Thavaneswaran A, Ruiz-Arruza I, Pellett F, Chandran $\mathrm{V}$, Cook RJ, et al. Minimal disease activity and anti-tumor necrosis factor therapy in psoriatic arthritis. Arthritis Care Res 2015; 67:842-7.

30. Husic R, Gretler J, Felber A, Graninger WB, Duftner C, Hermann J, et al. Disparity between ultrasound and clinical findings in psoriatic arthritis. Ann Rheum Dis 2014;73:1529-36.

31. Iervolino S, Di Minno MND, Peluso R, Lofrano M, Russolillo A, Di Minno G, et al. Predictors of early minimal disease activity in patients with psoriatic arthritis treated with tumor necrosis factor-a blockers. J Rheumatol 2012;39:568-73.

32. Janta I, Martínez-Estupiñán L, Valor L, Montoro M, Baniandres

Personal non-commercial use only. The Journal of Rheumatology Copyright $@$ (2020. All rights reserved 
Rodriguez O, Hernández Aragüés I, et al. Comparison between full and tapered dosages of biologic therapies in psoriatic arthritis patients: clinical and ultrasound assessment. Clin Rheumatol 2015;34:935-42.

33. Kalyoncu U, Erden A, Kilic L, Sari A, Armagan B, Karadag O, et al. DAPSA with twenty-eight joint counts may be useful for the assessment of psoriatic arthritis. Ann Rheum Dis 2016;75:1159-60.

34. Kavanaugh A, van der Heijde D, Beutler A, Gladman D, Mease P, Krueger GG, et al. Radiographic progression of patients with psoriatic arthritis who achieve minimal disease activity in response to golimumab therapy: results through 5 years of a randomized, placebo-controlled study. Arthritis Care Res 2016;68:267-74.

35. Kerr GS, Qaiyumi S, Richards J, Vahabzadeh-Monshie H, Kindred C, Whelton S, et al. Psoriasis and psoriatic arthritis in African-American patients - the need to measure disease burden. Clin Rheumatol 2015;34:1753-9.

36. Leung YY, Fong W, Lui NL, Thumboo J. Effect of ethnicity on disease activity and physical function in psoriatic arthritis in a multiethnic Asian population. Clin Rheumatol 2017;36:125-31.

37. Lubrano E, Perrotta FM, Parsons WJ, Marchesoni A. Patient's global assessment as an outcome measure for psoriatic arthritis in clinical practice: a surrogate for measuring low disease activity? J Rheumatol 2015;42:2332-8.

38. Lubrano E, Parsons WJ, Perrotta FM. Assessment of response to treatment, remission, and minimal disease activity in axial psoriatic arthritis treated with tumor necrosis factor inhibitors. J Rheumatol 2016;43:918-23.

39. Marin J, Felquer MLA, Garrot LF, Ruta S, Rosa J, Soriano ER. Patients with psoriatic arthritis fulfilling the minimal disease activity criteria do not have swollen and tender joints, but have active skin. J Rheumatol 2016;43:907-10.

40. Michelsen B, Diamantopoulos AP, Høiberg HK, Soldal DM, Kavanaugh A, Haugeberg G. Need for improvement in current treatment of psoriatic arthritis: study of an outpatient clinic population. J Rheumatol 2017;44:431-6.

41. Pappone N, Di Minno MND, Iervolino S, Lupoli R, Mader R, Zincarelli C, et al. The impact of concomitant diffuse idiopathic skeletal hyperostosis on the achievement of minimal disease activity in subjects with psoriatic arthritis. Rheumatol Int 2015;35:2041-66.

42. Perrotta FM, Marchesoni A, Lubrano E. Minimal disease activity and remission in psoriatic arthritis patients treated with anti-TNF- $\alpha$ drugs. J Rheumatol 2016;43:350-5.

43. Sheane BJ, Thavaneswaran A, Gladman DD, Chandran V. Attainment of minimal disease activity using methotrexate in psoriatic arthritis. J Rheumatol 2016;43:1718-23.

44. Theander E, Husmark T, Alenius GM, Larsson PT, Teleman A, Geijer M, et al. Early psoriatic arthritis: short symptom duration, male gender and preserved physical functioning at presentation predict favourable outcome at 5-year follow-up. Results from the Swedish Early Psoriatic Arthritis Register (SwePsA). Ann Rheum Dis 2014;73:407-13.

45. Mease P, Karki C, Etzel C, Kavanaugh A, Ritchlin CT, Malley W, et al. Clinical characteristics and disease outcomes in psoriatic arthritis patients by extent of body surface area affected by psoriasis: results from Corrona Registry. Arthritis Rheum 2015;67:3118.

46. Mease PJ, Karki C, Palmer JB, Etzel C, Kavanaugh A, Ritchlin $\mathrm{CT}$, et al. Clinical characteristics and disease activity in psoriatic arthritis patients with dactylitis or enthesitis: results from the Corrona Psoriatic Arthritis/Spondyloarthritis Registry. Arthritis Care Res 2017;69:1692-9.

47. Luime J, Tchetverikov I, Vis M, Appels C, van der Graaff W, Veris $\mathrm{J}$, et al. PASDAS, CPDAI and MDA evolution in the first 6 months after diagnosis of early psoriatic arthritis patients: results of the Depar Study Methods. Arthritis Rheum 2015;67:668.

48. Szentpetery A, Ikumi N, Kirby B, FitzGerald O. The presence of depression might be an important determinant of achieving minimal disease activity state in psoriatic arthritis. Arthritis Rheum 2016;68:1701.

49. Saldanha C, Zardin M, Reis A, Campos APB, Ribeiro SLE, Mancuso ACB, et al. Prevalence of minimal disease activity in "real life": cross sectional study in Brazilian patients with psoriatic arthritis and a literature review. Clin Exp Rheumatol 2016;34:752.

50. Mease P, Coates L, Kirkham B, McLeod LD, Mpofu S, Karyeakar $\mathrm{C}$, et al. Secukinumab improves minimal disease activity response rates in patients with active psoriatic arthritis: data from phase 3 FUTURE-2 study. Clin Exp Rheumatol 2016;34:777-8.

51. Lubrano E, De Socio A, Perrotta FM. Comparison of composite indices tailored for psoriatic arthritis treated with csDMARD and bDMARD: a cross-sectional analysis of a longitudinal cohort. J Rheumatol 2017;44:1159-64.

52. Coates L, Mease P, Husni M, Lespessailles E, Adams DH, Benichou $\mathrm{O}$, et al. Ixekizumab reduces disease activity in active psoriatic arthritis patients who had previous inadequate response to tumor necrosis factor-inhibitors. Ann Rheum Dis 2017;76:679.

53. Zabotti A, Idolazzi L, Quartuccio L, Sartori M, Fassio A, Zuliani F, et al. Identification of predictors of minimal disease activity in early psoriatic arthritis. Ann Rheum Dis 2017;76:940.

54. Mease P, Karki C, Liu M, Kavanaugh A, Ritchlin CT, Hunyh $\mathrm{DH}$, et al. Trends in clinical characteristics associated with achievement of minimal disease activity in response to biologic therapy in psoriatic arthritis - Analyses from the Corrona Psoriatic Arthritis/Spondyloarthritis (PSA/SPA) Registry. Ann Rheum Dis 2017;76:953

55. Vashisht P, Sayles H, Cannella A, Mikuls T, Michaud K. Generalizability of patients with rheumatoid arthritis in biologic agent clinical trials. Arthritis Care Res 2016;68:1478-88. 